\title{
Using the chemical noise background in MALDI mass spectrometry imaging for mass alignment and calibration Supplemental Information
}

\author{
Tobias Boskamp ${ }^{1,2^{*}}$, Delf Lachmund ${ }^{1}$, Rita Casadonte $^{3}$, Lena Hauberg-Lotte ${ }^{1}$, \\ Jan Hendrik Kobarg ${ }^{2}$, Jörg Kriegsmann ${ }^{3,4}$, and Peter Maass ${ }^{1,2}$ \\ ${ }^{1}$ Center for Industrial Mathematics, University of Bremen, Germany \\ ${ }^{2}$ SCiLS, Bremen, Germany \\ ${ }^{3}$ Proteopath, Trier, Germany \\ ${ }^{4}$ Center for Histology, Cytology and Molecular Diagnostic, Trier, Germany \\ *Corresponding author, tboskamp@uni-bremen.de
}

2019-11-25

\begin{abstract}
This is an appendix to the manuscript "Using the chemical noise background in MALDI mass spectrometry imaging for mass alignment and calibration" presenting a mass alignment and recalibration method that is specifically designed to operate on MALDI peptide imaging data. The proposed method exploits statistical properties of the characteristic chemical noise background observed in peptide imaging experiments. By comparing these properties to a theoretical peptide mass model, the effective mass shift of each spectrum is estimated and corrected.

This appendix includes a more formal presentation of the method, additional illustrative diagrams, as well as a detailed comparison to the previously published method by Ràfols et. al. 2018 .
\end{abstract}




\section{Appendix}

\section{A.1 Estimating mass shifts from local maxima}

Given a spectrum $S=\left(s_{j}, m_{j}\right)_{j=1 \ldots n}$ (typically a mean spectrum), consisting of the $n$ intensities $s_{1} \ldots s_{n}$ at $m / z$ locations $m_{1}<\ldots<m_{n}$, we denote by $T$ the local maxima in $S$, i.e. the set of those $m_{j}$ for which $s_{j}>\max \left\{s_{i}:|i-j|<u / 2\right\}$. The window width $u \in \mathbb{N}$ is chosen depending on the data $m / z$ resolution such that an interval smaller than $1 \mathrm{Da}$ is covered. Using the Kendrick shift $\delta_{\mathrm{av}}=\frac{m}{\lambda_{\mathrm{av}}}-\left\lfloor\frac{m}{\lambda_{\mathrm{av}}}+0.5\right\rfloor$ (see main text), we define the point cloud

$$
G(S)=\left\{\left(m, \delta_{\mathrm{av}}(m)\right): m \in T\right\},
$$

which is the graph of $\delta_{\text {av }}$ on $T$. The distribution of the points in $G$ is indicative of the mass distortion in $S$, as well as of the signal-to-noise ratio of the peptide background, as demonstrated by the examples shown in Fig. S-1.

To quantitatively analyze the properties of $G$, we define mass intervals $I_{k} \subset\left[m_{1}, m_{n}\right](k=1 \ldots K)$ covering the full mass range without overlap. Typically, one would choose equally sized intervals of length $\approx 100 \mathrm{Da}$. The local mass shift in interval $I_{k}$, denoted by $\Delta_{k}$, can then be estimated by taking the median of the respective $\delta_{\mathrm{av}}(m)$, and their dispersion, $w_{k}$, can be described using the interquartile range IQR. Thus, we define

$$
\begin{aligned}
& \Delta_{k}=\operatorname{median}\left\{\delta_{\mathrm{av}}(m): m \in T \cap I_{k}\right\}, \quad \text { and } \\
& w_{k}=\frac{1}{\sqrt{2} \operatorname{erf}^{-1}\left(\frac{1}{2}\right)} \operatorname{IQR}\left\{\delta_{\mathrm{av}}(m): m \in T \cap I_{k}\right\},
\end{aligned}
$$

where $\operatorname{erf}^{-1}$ denotes the inverse of the gaussian error function. The constant factor in (3) is chosen such that for normally distributed $\delta_{\mathrm{av}}(m)$, the corresponding $w_{k}$ equals twice the standard deviation, i.e. $\sim 95 \%$ of all values $\delta_{\text {av }}(m)$ lie within $\Delta_{k} \pm w_{k}$.

The values assumed for $w_{k}$ in real data depend on two factors: the variance $\sigma_{\mathrm{av}}^{2}$ of the true peptide masses around the cluster centers [2], and the variance $\sigma_{\text {bin }}^{2}$ induced by the binning of the $m / z$ axis.
Thus, we define the expected dispersion $v_{k}$ as

$$
\begin{aligned}
v_{k} & =\sqrt{\sigma_{\mathrm{av}}^{2}+\sigma_{\mathrm{bin}}^{2}}, \quad \text { with } \\
\sigma_{\mathrm{av}} & =0.02+2 \times 10^{-5} \bar{m}_{k}, \quad \sigma_{\mathrm{bin}}=b_{k} / \sqrt{12},
\end{aligned}
$$

where $\bar{m}_{k}$ denotes the center of interval $I_{k}$ and $b_{k}$ the average bin width in this interval.

If the observed dispersion $w_{k}$ significantly exceeds the expected dispersion $v_{k}$, this can have several reasons: First, the signal-to-noise ratio may be too low for the peptide background signal to be detectable. This effect is typically observed at the upper end of the $m / z$ range. Secondly, the spectrum may contain signals from molecules other than peptides, which is expected in the lower mass range. Finally, a large $w_{k}$ may indicate a strong mass misalignment within the set of spectra from which the mean spectrum is formed. In any of these cases, the quantity $\Delta_{k}$ is not accurate and reliable. Thus, as a rule of thumb, we consider mass shifts $\Delta_{k}$ valid only where $w_{k} / v_{k} \leq 1.2$.

\section{A.2 Estimating mass shifts from cir- cular moments}

An alternative approach to estimating the mass distortion of a spectrum is based on accumulating all spectral intensities in a $2 \mathrm{D}$ histogram over the $\mathrm{m} / \mathrm{z}$ and $\delta_{\mathrm{av}}$ axes. More specifically, in addition to the partition $\left(I_{k}\right)$ of the $m / z$ axis we consider a partition $\left(J_{l}\right)_{l=1 \ldots L}$ of the $\delta_{\mathrm{av}}$ axis $\left[-\frac{1}{2}, \frac{1}{2}\right] . \quad L=20$ equally sized intervals typically is a good choice. For a given spectrum $S=\left(s_{j}, m_{j}\right)_{j=1 \ldots n}$, let $\tilde{S}:\left[m_{1}, m_{n}\right] \rightarrow \mathbb{R}$ be a continuous interpolation of $S$, i.e. $\tilde{S}\left(m_{j}\right)=s_{j}$ for $j=1 \ldots n$. The aforementioned $2 \mathrm{D}$ histogram is represented by the matrix $H=\left(H_{k, l}\right)$ with

$$
H_{k, l}=\frac{1}{\int_{I_{k}} \tilde{S}(t) d t} \int_{\Gamma_{k, l}} \tilde{S}(t) d t
$$

where $\Gamma_{k, l}=\left\{m \in I_{k}: \delta_{\mathrm{av}}(m) \in J_{l}\right\}$ is the subset of the $m / z$ range associated with the $2 \mathrm{D}$ bin indexed by $(k, l)$ (see Fig. S-2, as well as main text, Fig. 2).

Given the $2 \mathrm{D}$ histrogram $H$, we obtain an estimate for the local mass shift $\Delta_{k}$ by localizing the center of gravity of columns of $H$. Here, we need to take into 
account the circular structure of the $\delta_{\mathrm{av}}$ axis, i.e. the fact that the lines $\delta_{\mathrm{av}}=\frac{1}{2}$ and $\delta_{\mathrm{av}}=-\frac{1}{2}$ are identified with each other. Thus, we compute the first circular moments of columns of $H$,

$$
\mu_{k}=\sum_{l=1}^{L} H_{k, l} e^{2 \pi i \bar{\delta}_{l}}
$$

where $\bar{\delta}_{l}$ denotes the center of interval $J_{l}$. Since from (5) it follows that $\sum_{l=1}^{L} H_{k, l}=1$, the $\mu_{k}$ are complex quantities with $\left|\mu_{k}\right| \leq 1$. The local mass shifts $\Delta_{k}$ are related to their complex arguments by

$$
\Delta_{k}=\frac{1}{2 \pi} \arg \left(\mu_{k}\right)
$$

whereas the absolute values $\left|\mu_{k}\right|$ provide information about the dispersion of the accumulated intensities in the columns of $H$.

The computation of the $\mu_{k}$ can be further simplified by considering the limit case of an infinitesimally fine partition $J$, i.e. $L \rightarrow \infty$ and $\sup \left|J_{l}\right| \rightarrow 0$. This leads to

$$
\mu_{k}=\frac{1}{\int_{I_{k}} \tilde{S}(t) d t} \int_{I_{k}} \tilde{S}(t) e^{2 \pi i t / \lambda_{\mathrm{av}}} d t,
$$

which can be obtained without computing $H$. For a numerical evaluation, a suitable quadrature rule may be chosen, and the $m_{j}$ can be used as sampling points. Since $\tilde{S}\left(m_{j}\right)=s_{j}$, this avoids the need to explicitly interpolate the discrete spectrum $S$. In the most simple case of approximating (8) by means of Riemann sums, we obtain

$$
\mu_{k}=\frac{1}{\sum_{j \in N_{k}} s_{j}} \sum_{j \in N_{k}} s_{j} e^{2 \pi i m_{j} / \lambda_{\mathrm{av}}},
$$

where $N_{k}=\left\{j \in\{1 \ldots n\}: m_{j} \in I_{k}\right\}$ denotes the set of indices $j$ with $m_{j}$ falling in interval $I_{k}$.

\section{A.3 Comparison to Ràfols 2018}

For a comparison to the method of Ràfols et. al. [1], one of the more recent alignment methods applicable to FFPE peptide imaging data, we used the implementation available as part of the rMSIproc package ${ }^{1}$.

\footnotetext{
${ }^{1}$ https://github.com/prafols/rMSIproc
}

We applied this algorithm to the example tissue section used in the main text (dataset no. 21, see main text, Fig. 3), and used the same methods for assessing relative mass alignment and absolute mass accuracy on the aligned output data.

As regards the computational complexity, algorithmic processing times of the rMSIproc implementation could not be measured separately from disk $\mathrm{I} / \mathrm{O}$ times. Therefore, we performed the experiments on a mobile workstation (Intel i7-8550U CPU @ $1.80 \mathrm{GHz}$, 32 GB RAM) with the data read from a fast SSD drive to minimize the impact of disk $\mathrm{I} / \mathrm{O}$ on the execution speed. For a fair comparison, we also included the times to load and store the spectral data in the measurements for our proposed method (peptide background alignment, PBA). A summary of the results is given in the table below, more details are found in Table S-1.

\begin{tabular}{lrc}
\hline & PBA & rMSIproc \\
\hline Relative alignment & (ppm) & \\
Mean spectrum peaks & 12.4 & 11.2 \\
Reference peaks & 12.4 & 10.0 \\
\hline Absolute accuracy & $(\mathbf{p p m})$ & \\
Reference peaks & -27.5 & 161.3 \\
\hline Execution times $(\mathbf{s e c})$ & \\
Processing & 44.4 & \\
Data I/O & 164.4 & \\
Total & 208.8 & 890.9 \\
\hline
\end{tabular}

As can be seen, both methods achieve similar results with respect to relative mass alignment. The rMSIproc algorithm is not designed to align to an apriori reference, and hence is not able to increase the absolute mass accuracy in the data. As regards execution speed, the rMSIproc method needs to process the spectral data twice, once for selecting a reference spectrum, and a second time for performing the alignment. Thus, we can estimate the data $\mathrm{I} / \mathrm{O}$ time to be in the range of 250-330 sec (between 1.5 and 2 times the data $\mathrm{I} / \mathrm{O}$ time for $\mathrm{PBA}$ ), indicating an algorithmic processing time between 560 and $640 \mathrm{sec}$. 


\section{References}

[1] P. Ràfols, E. del Castillo, O. Yanes, J. Brezmes, and X. Correig. Novel automated workflow for spectral alignment and mass calibration in ms imaging using a sputtered ag nanolayer. Analytica Chimica Acta, 1022:61-69, 2018.

[2] W. E. Wolski, M. Farrow, A. K. Emde, H. Lehrach, M. Lalowski, and K. Reinert. Analytical model of peptide mass cluster centres with applications. Proteome Sci, 4:18, 2006. 

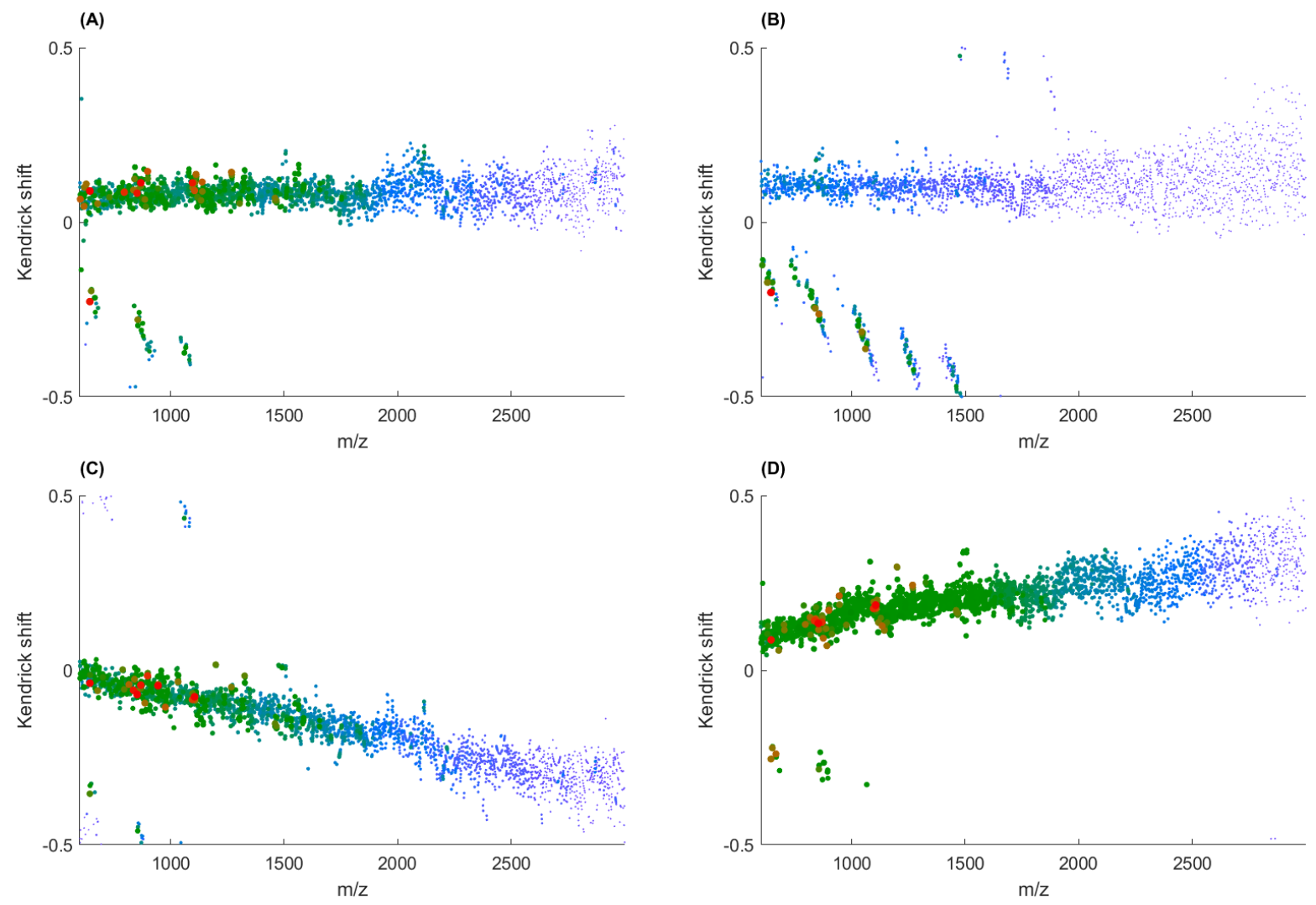

Figure S-1: Kendrick shift plots for four different MALDI MSI datasets reveal characteristic spectral data properties: (A) High signal-to-noise ratio and low mass distortion ( $<0.1 \mathrm{Da}$ ) across the full $\mathrm{m} / z$ range. (B) Low signal-to-noise ratio, noticeable by the widening of the peptide point cloud beyond 1800 Da. (C) High mass shift towards lower masses, as much as $-0.25 \mathrm{Da}(-100 \mathrm{ppm})$ at $m / z 2500$. (D) High mass shift towards higher masses, approx. $0.3 \mathrm{Da}(120 \mathrm{ppm})$ at $\mathrm{m} / z$ 2500. Dot sizes and colors indicate signal intensities, from blue $=$ low to red $=$ high . 

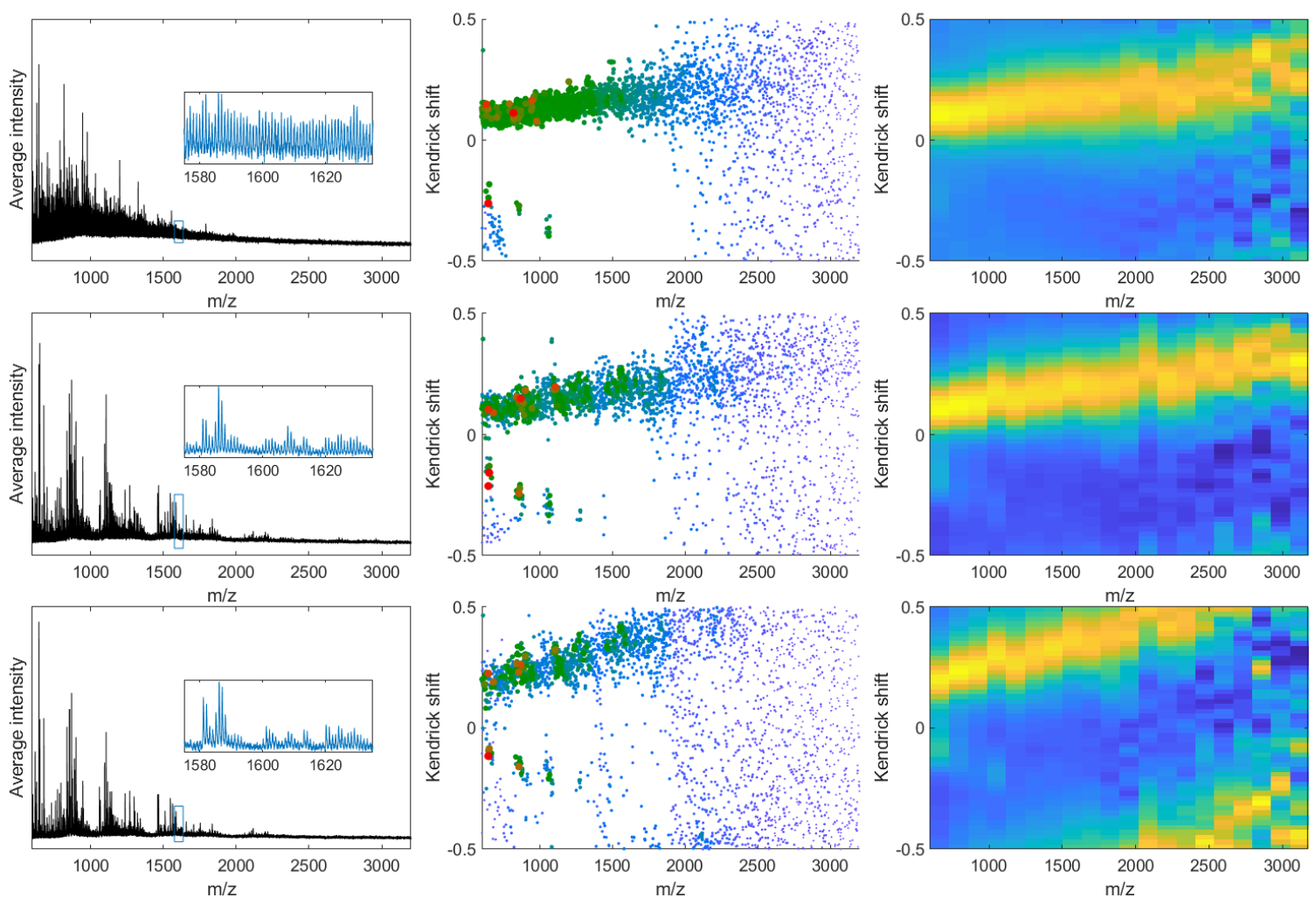

Figure S-2: Comparison of three exemplary single spectra (left column) taken from dataset no. 21 (cf. Table S-1). Due to the lower signal-to-noise ratio, the Kendrick shift plots obtained from local maxima reflect the peptide background only up to about $2000 \mathrm{Da}$ (middle). In the Kendrick shift histograms, however, it is observable over the full mass range (right). Note the "wrap around" occuring in the third example around $2500 \mathrm{Da}$ where the local mass shift exceeds 0.5 Da (bottom right). 
(A)

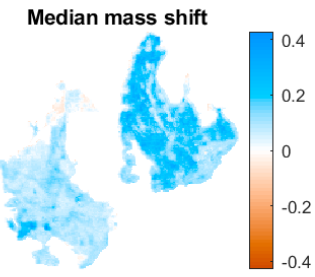

(B)

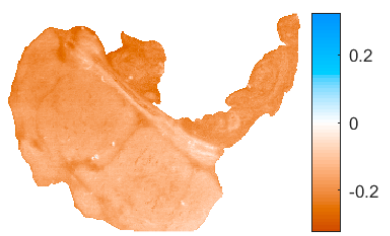

(C)

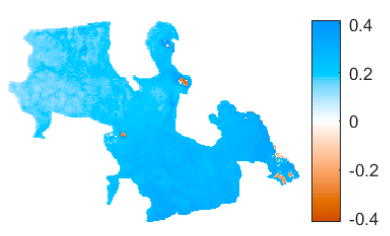

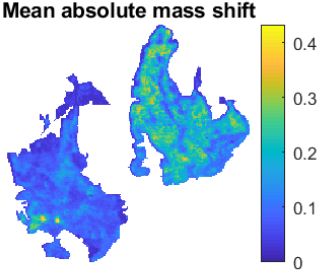
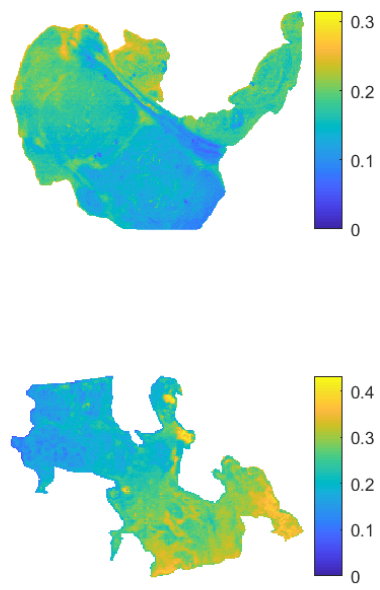
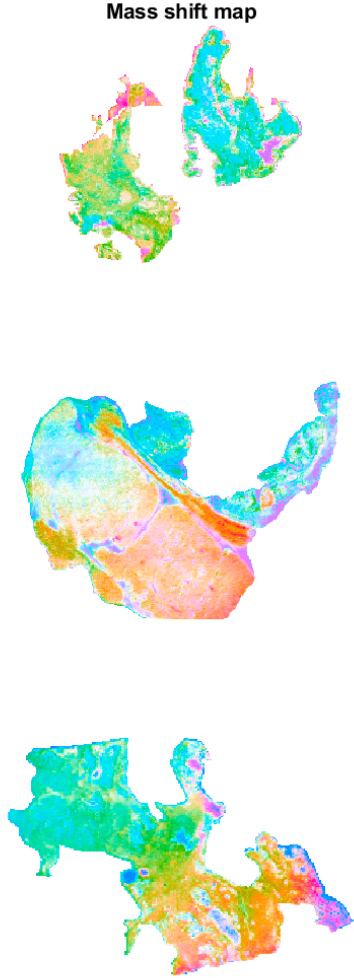

Figure S-3: Comparison of different mass shift profile visualizations. Left: Median mass shift across full $\mathrm{m} / z$ range. Middle: Mean absolute mass shift across $m / z$ range. Right: Color encoded mass shift map from first two PCA components. Rows (A), (B), and (C) correspond to datasets no. 4, 7, and 21, resp. (cf. Table S-1). 

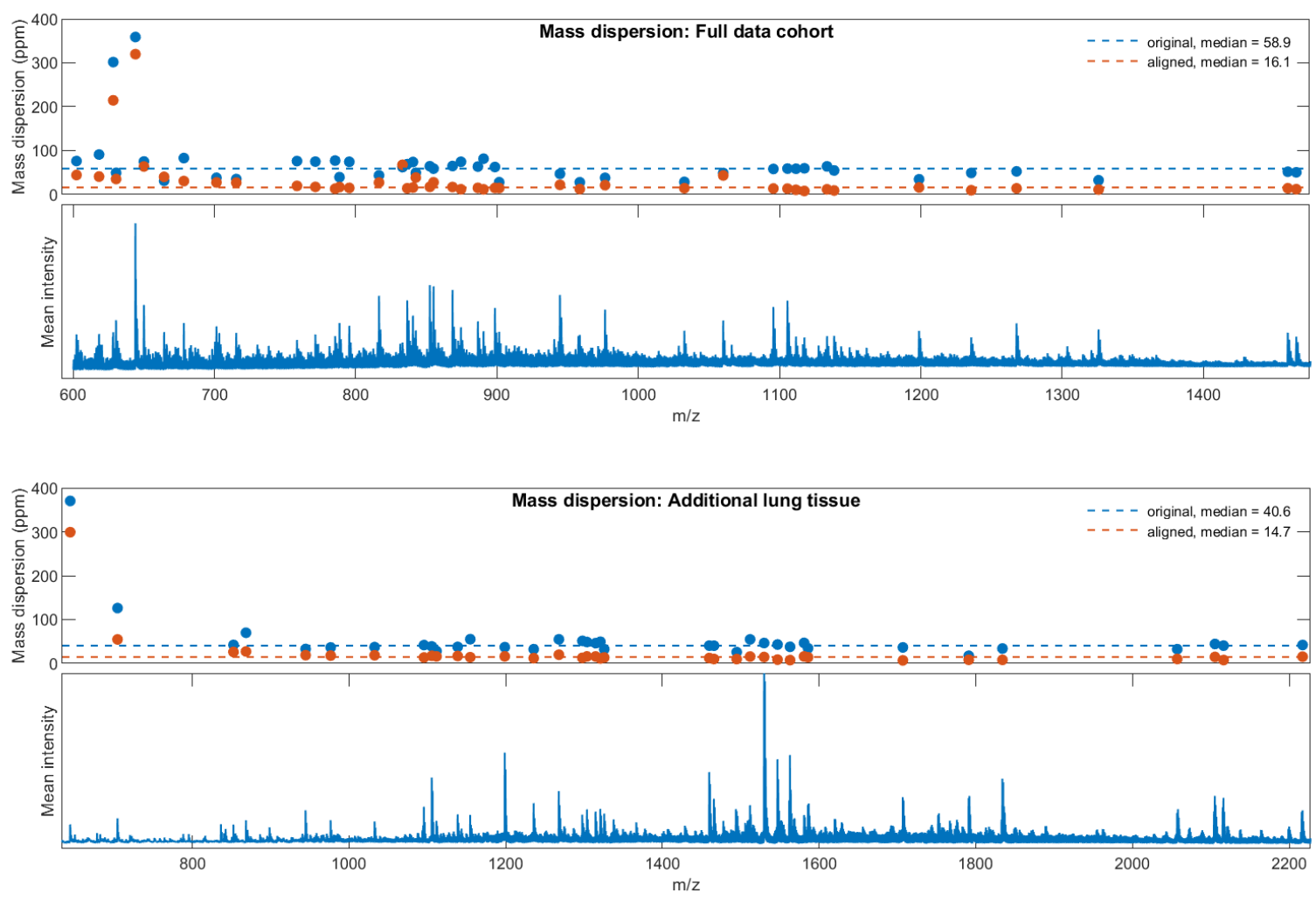

Figure S-4: Top: Overall mean spectrum, as well as mass dispersion of major peaks before and after alignment across all 31 datasets. Bottom: Mean spectrum and mass dispersion of major peaks before and after alignment for an additional lung tissue section (Fig. S-5). 


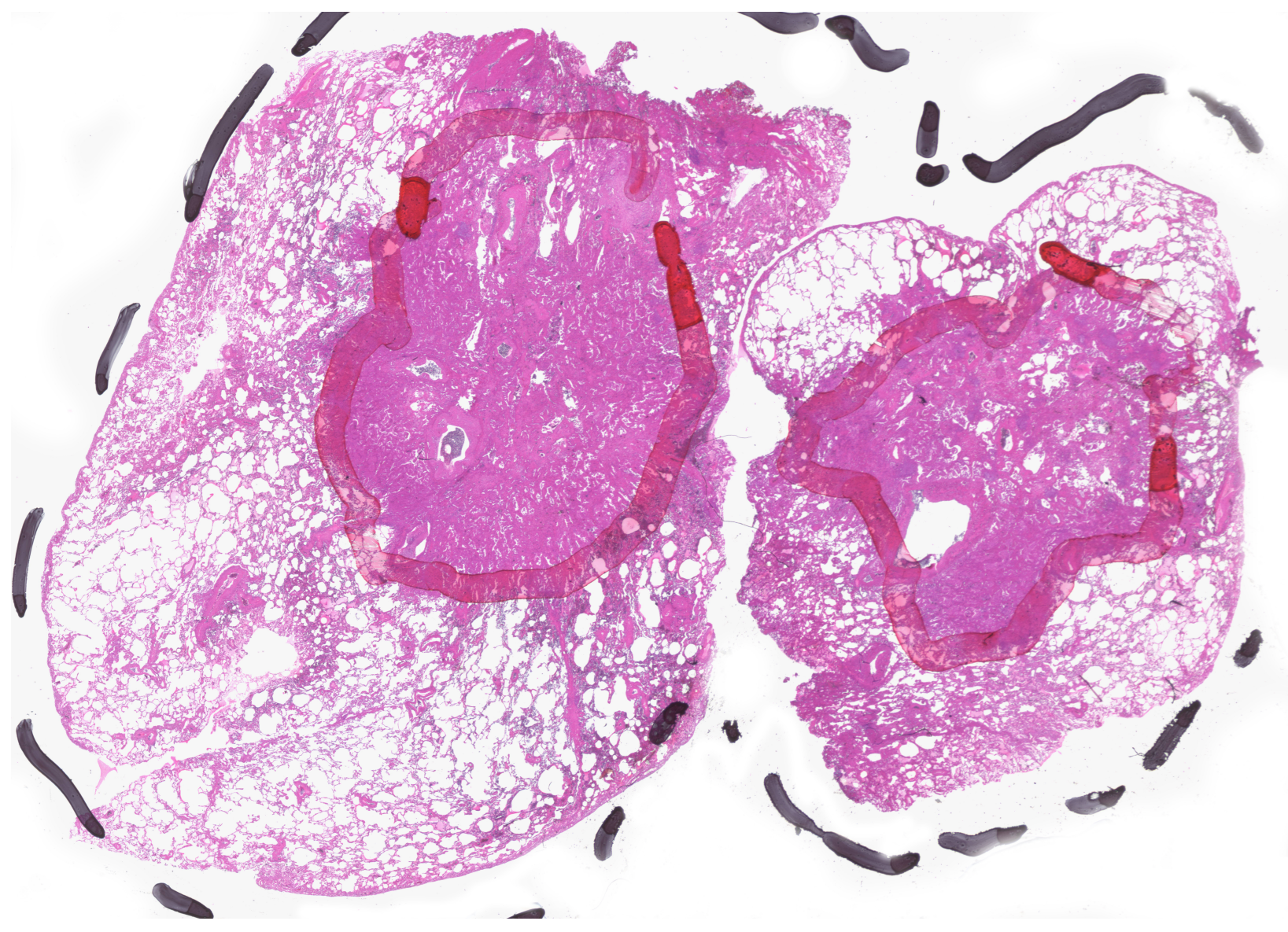

Figure S-5: HE scan of the additional lung tissue section used for Fig. S-4 (bottom), showing a lung tumor (red markup) embedded in normal lung parenchyma. 